\title{
NOUVELle
}

\section{Les rétrovirus foamy zoonotiques : une première étude médicale chez les personnes infectées}

Florence Buseyne
Unité d'épidémiologie et physiopathologie des virus oncogènes, Institut Pasteur, 28, rue du Docteur Roux, 75015 Paris, France.

florence.buseyne@pasteur.fr
> Les émergences infectieuses zoonotiques sont un enjeu majeur de santé publique [1-3] $(\rightarrow)$.

Deux familles de rétrovirus ont émergé dans la population humaine depuis un réservoir simien : les virus de l'immunodéficience humaine (VIH) et les virus humains T lymphotropes (human $T$ lymphotropic virus, HTLV). La pandémie du VIH est une des plus récentes et des plus importantes de l'histoire [4] $(\rightarrow)$ Voir la Synthèse de M. Peeters et al., $\mathrm{m} / \mathrm{s}$ $n^{\circ}$ 6-7, juin-juillet 2008 page 621

$(\rightarrow)$.

Une troisième famille de rétrovirus infecte l'homme : les virus foamy simiens (VFS)

Les virus foamy simiens (VFS) sont des rétrovirus complexes (Figure 1A) [5]. Leur cycle de réplication partage des propriétés avec celui des orthorétrovirus (intégration) et celui des hépadnavirus (transcription inverse tardive). Leur tropisme cellulaire est ubiquitaire et leur récepteur inconnu. In vitro, leur effet cytopathogène donne un aspect mousseux aux cellules infectées (Figure 1B). Cette caractéristique fut retenue pour les nommer: spumarétrovirus ou «foamy virus ».

L'homme n'est pas un hôte naturel des VFS, mais peut être infecté de façon persistante à la suite d'une exposition aux fluides d'animaux infectés, principalement par morsure $[5,6]$. En effet, les VFS se répliquent dans la cavité buccale des primates non humains (PNH). La très grande majorité des personnes infectées ont une exposition démontrée aux VFS ; elles sont donc les premiers hôtes d'un rétrovirus simien zoonotique ${ }^{1}$.

\section{L'exposition et l'infection} par les VFS est un phénomène actuel dont les conséquences pour la santé humaine sont inconnues

Les infections par des VFS zoonotiques ont été rapportées dans les pays industrialisés à partir de 1995, puis en Afrique, en Asie et en Amérique du Sud [5, 6]. Ces contaminations ont été montrées dans des contextes variés: professionnels, chasse, dépeçage et consommation de viande «de brousse », contact avec des animaux commensaux, en particulier sur les sites religieux d'Asie (Figure 2). Ainsi, le risque de contamination suite à la visite d'un de ces sites religieux a été estimé à 6/1000 [7].

Si la transmission des VFS aux hommes est aujourd'hui établie, les conséquences cliniques ont été très peu étudiées. Ces virus établissent des infections persistantes, avec présence du génome viral et du virus compétent pour la réplication dans les cellules sanguines. Cette infection est décrite comme « apparemment asymptomatique » chez l'homme et chez les primates non humains. La majorité

${ }^{1}$ Avec transmission entre animal et homme ; l'animal étant généralement le réservoir. des personnes infectées vit dans des régions où les structures de surveillance et de soins sont modestes ou inexistantes. Dans les pays développés, les premiers cas décrits mentionnaient le bon état de santé des personnes infectées. Une seule étude longitudinale a été menée aux États-Unis. Pendant cinq ans, sept personnes ont été suivies annuellement par un entretien téléphonique portant sur leur état de santé et un bilan hématologique. Cette étude n'a pas rapporté la survenue d'événement médical notable, mais aucun témoin non infecté n'a été suivi dans cette étude.

\section{Une étude cas-contrôle de chasseurs d'Afrique centrale}

Nous avons étudié des chasseurs vivant dans des zones rurales du sud Cameroun, personnes que nous avions testées pour l'infection par les virus foamy simiens à l'occasion de grandes enquêtes épidémiologiques $[8,9]$. Pour cela, nous avons bâti une étude cas-contrôle ${ }^{2}$ portant sur 24 cas diagnostiqués infectés par un VFS sur la base d'un test sérologique et de sa confirmation moléculaire. Ils ont été appariés à 24 contrôles, des hommes pratiquant la chasse, de même âge et vivant dans le même village ou un village voisin. Ces sujets, âgés de 22 à 67 ans, étaient en bonne santé apparente.

\footnotetext{
2 Une étude cas-contrôle (ou cas-témoin) est une étude clinique comparant deux groupes de sujets :

- les cas présentant un événement donné (ici, l'infection par un VFS) ;

- les contrôles ne présentant pas l'événement donné, mais qui sont par ailleurs les plus semblables que possible aux cas.
} 


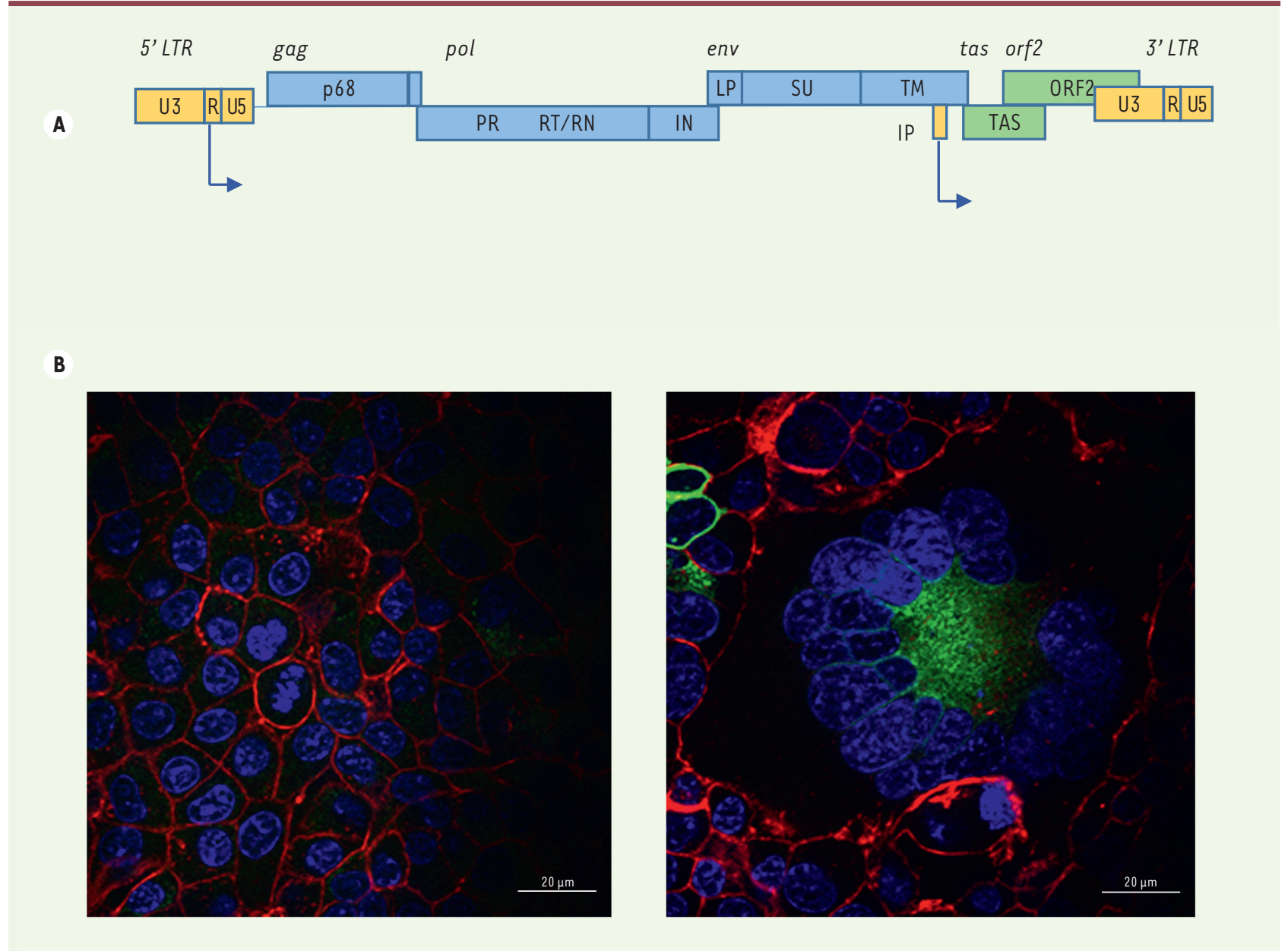

Figure 1. Les virus foamy : génome et effet cytopathogène. A. Représentation schématique du génome des virus foamy simiens (VFS). Les noms des gènes et des régions régulatrices sont en italique. Les protéines sont indiquées dans les boîtes. Les flèches correspondent aux deux sites d'initiation de la transcription. Les régions régulatrices sont indiquées en jaune, celles codant les protéines structurales sont en bleu, et celles codant le transactivateur Tas en vert. L'épissage des ARN messagers tas et orf2 permet la synthèse d'une cinquième protéine virale, Bet (Between env and LTR). LTR : long terminal repeat; PR : protéase ; RT : transcriptase inverse ; RN : ribonucléase ; LP : leader peptide ; SU : surface antigen ; TM : transmembrane protein; ORF : open reading frame; IP : internal promotor. B. Cellules de rein de hamster (lignée BHK-21) non infectées (partie gauche) et infectées (partie droite) par un VFS et marquées par du DAPI (noyaux en bleu), un anticorps spécifique de l'enveloppe virale (en vert), et un marqueur de l'actine filamenteuse : la phallö̈dine (en rouge). Un effet cytopathogène caractéristique est observé.

Ils ont été transportés jusqu'à Yaoundé où un examen clinique et un bilan sanguin ont été réalisés $[10]$. Ces examens couvraient les principales fonctions physiologiques, sans hypothèse a priori, faute de toute donnée préliminaire.

Dans l'ensemble de la population étudiée, des signes de pathologies ont été répertoriés. II s'agissait de signes généraux avec prédominance d'adénopathies (chez $38 \%$ des sujets), de signes cutanés (38\%), de signes gastro-intestinaux (21\%), de signes cardiorespiratoires ( $50 \%$, en par- ticulier une pression artérielle élevée) et de signes neurologiques (8\%). L'analyse a montré la même fréquence de ces signes cliniques chez les cas et les contrôles, que I'on considère les signes individuellement, par organe affecté, ou leur nombre total. La prévalence élevée de signes cliniques observés impose donc une conclusion prudente concernant les conséquences de l'infection par un VFS. Néanmoins, l'absence de signes cliniques significativement associés à l'infection est rassurante pour ces personnes. $\varepsilon$ n revanche, les bilans sanguins des cas et des contrôles présentaient des niveaux différents de marqueurs érythrocytaires : la concentration d'hémoglobine, l'hématocrite et le volume globulaire moyen étaient significativement plus faibles chez les cas que chez les contrôles. Pour l'hémoglobine, les valeurs médianes étaient respectivement de 12,7 et $14,4 \mathrm{~g} / \mathrm{dl}$. Sur la base des normes de l'Organisation mondiale de la santé (OMS), $58 \%$ des cas VFS, contre seulement $17 \%$ chez les 


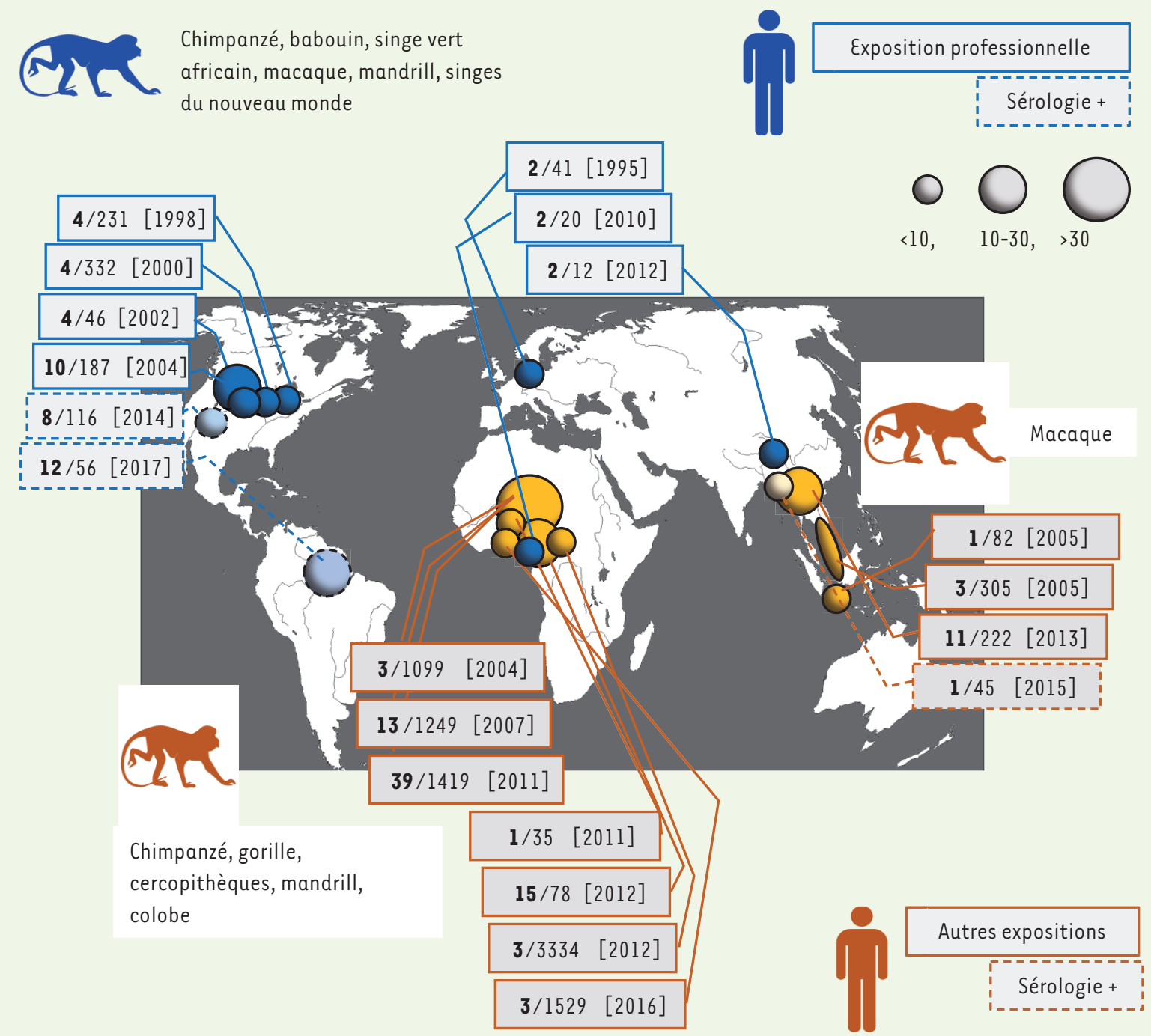

Figure 2. Récapitulatif des cas documentés d'infection par un virus foamy simiens (VFS). Les études ont été publiées dans deux revues [5, 6]. Chaque étude est représentée par un cercle dont la taille dépend du nombre de cas décrits. Les boîtes indiquent le nombre de personnes positives (en gras), le nombre de personnes testées et l'année de publication. Les contaminations en milieu professionnel sont indiquées en bleu et celles dans d'autres contextes en orange. Les trois études pour lesquelles les tests sérologiques étaient positifs et les tests moléculaires négatifs sont indiquées par des pointillés. Les espèces de primates non-humains (PNH) hôtes des VFS transmis sont précisées pour chaque contexte d'exposition.

contrôles, souffraient d'anémie légère ou modérée.

Les populations de globules blancs étaient similaires dans les deux groupes, à l'exception de niveaux plus faibles de basophiles. Les concentrations de plusieurs marqueurs biochimiques différaient entre les deux groupes et plus particulièrement ceux associés à la fonction rénale : les concentrations d'urée, de créatinine et de protéines étaient plus élevées chez les cas que chez les contrôles.
Nous avons vérifié l'absence de biais lié aux agents infectieux les plus courants, tels HTLV-1 et HBV (virus de l'hépatite B). La comparaison des deux groupes a montré des niveaux de bilirubine plus bas chez les individus infectés ; ce marqueur de lyse érythrocytaire exclut donc une hémolyse possiblement induite par des parasites. Les niveaux équivalents de lymphocytes, d'éosinophiles et de neutrophiles sont également des indicateurs d'une exposition infectieuse similaire pour les deux groupes.
Nous avons recherché des indicateurs sur les causes possibles de la baisse du niveau d'hémoglobine, en utilisant des plasmas congelés. Par électrophorèse des protéines, nous avons pu, par exemple, exclure l'existence d'une gammapathie ${ }^{3}$. En prenant en compte l'ensemble des marqueurs testés, deux hypothèses

\footnotetext{
3 Gammapathie ou dysglobulinémie désigne une anomalie se traduisant par un excès d'une immunoglobuline monoclonale dans le sérum et/ou les urines.
} 
peuvent être proposées : la première est celle d'une anémie inflammatoire; la seconde serait un défaut de l'érythropoïèse ou de sa régulation. Notons également des pistes qui n'ont pas été explorées, comme l'étude de polymorphismes génétiques associés au système érythropoïétique qui pourraient influencer la susceptibilité à l'infection par les VFS.

\section{Limites et conclusions}

Les limites de ce travail sont multiples. Nos résultats ont été obtenus par l'étude de sujets sains : il en résulte donc un biais majeur pour conclure sur une association entre infection par un virus foamy simiens et conséquences médicales. Les limites sont également liées au découplage entre le lieu du risque infectieux et celui de la structure qui l'étudie [1]. Les personnes participant à l'étude vivent à une journée de route de Yaoundé, la capitale du Cameroun, où les examens cliniques et les analyses biologiques ont été réalisés. Cela engendre une logistique lourde à gérer par l'équipe de recherche, qui limite les possibilités d'investigation. La mise en place d'études depuis la primo-infection est impossible : la plupart des sujets victimes de morsures ne sont pas hospitalisés et ont recours à des soins traditionnels. Une autre limite de notre étude est l'impossibilité d'analyser le réser- voir naturel, principalement les gorilles, espèce protégée et menacée d'extinction. Nous avons observé un spectre de différences entre les marqueurs cellulaires et biochimiques du sang, dont une baisse du niveau d'hémoglobine qui est médicalement significative. Une association statistique entre l'infection par un VFS et ces altérations hématologiques ne démontre pas que le virus en soit la cause directe. Cependant, une relation causale est biologiquement plausible: les VFS sont des inducteurs puissants des interférons de type I et pourraient provoquer une anémie inflammatoire; ils infectent également tous les types cellulaires dont les cellules rénales et les précurseurs érythroïdes.

$\varepsilon n$ conclusion, les virus foamy simiens sont responsables de zoonoses fréquentes. Nous avons montré qu'ils ne seraient pas silencieux sur le plan physiologique. Un risque pour la santé humaine ne peut donc être exclu et mérite une veille chez les populations exposées. $\diamond$

Zoonotic foamy viruses: first medical study of infected people

\section{REMERCIEMENTS}

F. Buseyne remercie tous les coauteurs de la publication originale. Mathilde Couteaudier est l'auteure des photos présentées. Le travail a été soutenu par l'Institut Pasteur (Programme Transversal de Recherche, PTR437), l'Agence National de la Recherche (projet REEMFOAMY, ANR 15-CE-150008-01) et le laboratoire d'excellence biologie intégrative des maladies émergentes ( $L a b \varepsilon x$ IBEID, ANR 10-LABX-62-IBEID).

\section{LIENS D'INTÉRÊT}

L'auteure déclare n'avoir aucun lien d'intérêt concernant les données publiées dans cet article.

\section{RÉFÉRENCES}

1. Flahault $A$. Les trois paradoxes du risque épidémique. Med Sci (Paris) 2014 ; $30: 823-4$.

2. Cle $M$, Salinas $S$, Lecollinet $S$, et al. Le virus Usutu : Ia menace fantôme. Med Sci (Paris) 2018 ; 34 : 709-16.

3. Loustalot F, Creyssels S, Salinas S, et al. Les adénovirus non-humains : un risque zoonotique? Med Sci (Paris) $2015 ; 31: 1102-8$.

4. Peeters M, Chaix ML, Delaporte $\varepsilon$. Phylogénie des SIV et des VIH. Med Sci (Paris) $2008 ; 24: 621-8$.

5. Pinto-Santini DM, Stenbak CR, Linial ML. Foamy virus zoonotic infections. Retrovirology $2017 ; 14: 55$.

6. Gessain A, Rua R, Betsem $\varepsilon$, et al. HTLV-3/4 and simian foamy retroviruses in humans : Discovery, epidemiology, cross-species transmission and molecular virology. Virology 2013 ; 435 : 187-99.

7. Engel G, Hungerford LL, Jones-Engel L, et al. Risk assessment : a model for predicting cross-species transmission of simian foamy virus from macaques (M-fascicularis) to humans at a monkey temple in Bali, Indonesia. Am J Primatol 2006; 68: 934-48.

8. Calattini S, Betsem EBA, Froment A, et al. Simian foamy virus transmission from apes to humans, rural Cameroon. Emerg Infect Dis 2007 ; 13 : 1314-20.

9. Betsem $\varepsilon$, Rua R, Tortevoye P, et al. Frequent and recent human acquisition of simian foamy viruses through apes' bites in Central Africa. PLoS Pathog 2011 ; 7 : e1002306.

10. Buseyne F, Betsem $\varepsilon$, Montange T, et al. Clinical signs and blood test results among humans infected with zoonotic simian foamy virus : a case-control study. J Infect Dis $2018 ; 218$ : 144-51

\section{AMPS (Association Médecine Pharmacie Sciences)}

La période de candidature pour la passerelle permettant d'intégrer directement les études de Santé (Médecine, Pharmacie, Odontologie et Maïeutique) après un diplôme de niveau Bac +5 ou plus approche à grand pas. Pour cette occasion, l'AMPS (Association Médecine Pharmacie Sciences) a le plaisir de vous présenter son guide de candidature au concours passerelle, ainsi que son guide pour préparer sa rentrée directement en $2^{\mathrm{e}}$ ou $3^{\mathrm{e}}$ année des études de Santé, rédigé par des étudiants qui s'y trouvent déjà

Si vous êtes un candidat potentiel, ou que vous en connaissez, n’hésitez pas à les utiliser, ils contiennent théoriquement toutes les informations utiles pour passer du la candidature à la rentrée dans l'année supérieure. Et n'hésitez pas non plus à nous faire part de toutes vos remarques concernant ces guides, nous nous ferons un plaisir de les améliorer.

Enfin, si vous-même avez bénéficié de cette passerelle par le passé, n’hésitez pas à nous contacter pour nous faire part de vos témoignages, vos successeurs vous en remercieront grandement.

Vous trouverez toutes les informations intéressantes sur le site de l'AMPS: https://amps-asso.org/ > Opportunités > Guides Yanis Bendjelal, rédacteur en chef des guides Sciences-Santé de l'AMPS 